\title{
Multiplying logical values ${ }^{1}$
}

\author{
Grzegorz Malinowski
}

\begin{abstract}
The modern history of many-valuedness starts with Łukasiewicz's construction of three-valued logic. This pioneering, philosophically motivated and matrix based construction, first presented in 1918, was in 1922 extended to $n$-valued cases, including two infinite ones. Soon several constructions of many-valued logic appeared and the history of the topic became rich and interesting. However, as it is widely known, the problem of interpretation of multiple values is still among vexed questions of contemporary logic.

With the paper, which essentially groups my earlier settlements, from [3], [4], [7] and [8], I intend to put a new thread into discussion on the nature of logical many-valuedness. The topics, touched upon, are: matrices, tautological and non-tautological many-valuedness, Tarski's structural consequence and the Lindenbaum-Wójcicki completeness result, which supports the Suszko's claim on logical two-valuedness of any structural logic. Consequently, two facets of many-valuedness referential and inferential - are unravelled. The first, fits the standard approach and it results in multiplication of semantic correlates of sentences, and not logical values in a proper sense. The second many-valuedness is a metalogical property of inference and refers to partition of the matrix universe into more than two disjoint subsets, used in the definition of inference.
\end{abstract}

Keywords: three-valued logic, many-valuedness, matrix, tautology, consequence operation, structurality, logical two-valuedness, Suszko's Thesis, non-fregean logic, logical three-valuedness, inferential many-valuedness, inferential values.

\section{Tautological many-valuedness}

A referential construction of a many-valued logic starts with the choice of the sentential language $L$, i.e. an algebra $L=\left(F o r, F_{1}, \ldots\right.$, $\left.F_{m}\right)$ freely generated by the set of variables $\operatorname{Var}=\{p, q, r, \ldots\}$. Formulas, i.e. elements of For, are then built from variables using

\footnotetext{
${ }^{1}$ The paper is an extended version of the invited lecture to the international conference "7th Smirnov's Readings in Logic" June 22-24, 2011, Moscow (Russia).
} 
the operations $F_{1}, \ldots, F_{m}$ representing the sentential connectives. In most cases, either the language of the classical sentential logic

$$
L_{k}=(\text { For }, \neg, \rightarrow, \vee, \wedge, \leftrightarrow)
$$

with negation $(\neg)$, implication $(\rightarrow)$, disjunction $(\vee)$, conjunction $(\wedge)$, and equivalence $(\leftrightarrow)$, or some of its reducts is considered. Subsequently, one defines an algebra $A$ similar to $L$ and chooses a non-empty subset $D$ of designated elements of the universe of $A$. Any such interpretation structure

$$
M=(A, D)
$$

is called logical matrix.

Traditionally, the system of sentential logic is defined as the set of all formulas taking for every valuation $h$ (a homomorphism) of $L$ in $M$

$$
\mathrm{E}(M)=\{\alpha \in \text { For }: \text { for every } h \in \operatorname{Hom}(L, A), h(\alpha) \in D\},
$$

called the content of $M$. Thus, the classical matrix based on $\{0,1\}$ and the connectives defined by the classical truth-tables has the form of

$$
M_{2}=(\{0,1\}, \neg, \rightarrow, \vee, \wedge, \leftrightarrow,\{1\})
$$

Obviously, then $\mathrm{E}\left(M_{2}\right)=T A U T$, the set of tautologies. That is why we shall also sometimes refer to $\mathrm{E}(M)$ as the set of tautologies of $M$ even if the matrix $M$ is not classical.

If the content of a multiple-element matrix $M$ does not coincide with $T A U T, \mathrm{E}(M) \neq T A U T$, we say that $M$ defines tautologically many-valued logic. The best known example of this kind is the historically first construction by Eukasiewicz's in [2], here presented as

$$
M_{3}=(\{0,1 / 2,1\}, \neg, \rightarrow, \vee, \wedge, \leftrightarrow,\{1\})
$$

with the connectives defined by the tables:

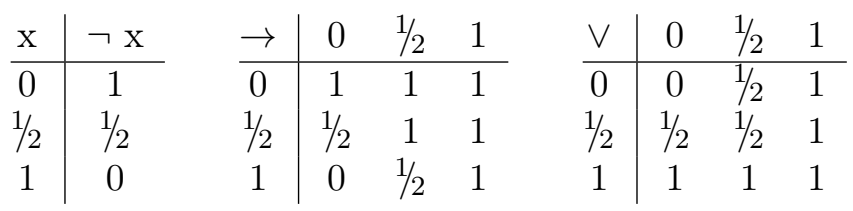




\begin{tabular}{|c|c|c|c|c|c|c|c|}
\hline & 0 & $1 / 2$ & 1 & $\leftrightarrow$ & 0 & $1 / 2$ & 1 \\
\hline 0 & 0 & 0 & 0 & $\overline{0}$ & 1 & & 0 \\
\hline $1 / 2$ & 0 & $1 / 2$ & $1 / 2$ & $1 / 2$ & $1 / 2$ & & $1 / 2$ \\
\hline 1 & 0 & $1 / 2$ & 1 & 1 & 0 & & 1 \\
\hline
\end{tabular}

Since e.g. $p \vee \neg p$ and $\neg(p \wedge \neg p)$ take the value $1 / 2$ when $p$ takes $1 / 2$, and thus they are not Eukasiewicz tautologies, $\mathrm{E}\left(M_{3}\right) \neq T A U T$.

Subsequently, to see that the separate multiplication of values of the base matrix is not sufficient for getting many-valued logic, consider the following three-element matrix, cf. [7],

$$
\begin{aligned}
& N_{3}=(\{0, t, 1\}, \neg, \rightarrow, \vee, \wedge, \leftrightarrow,\{t, 1\}),
\end{aligned}
$$

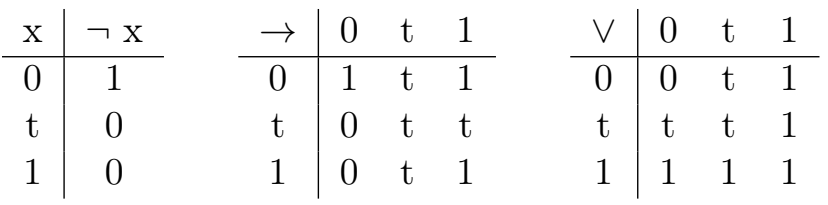

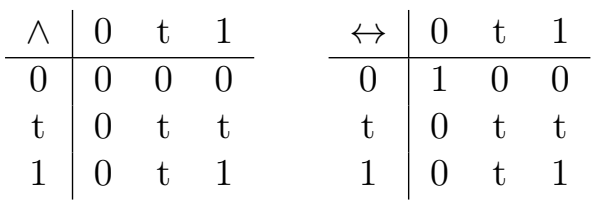

It is easy to show, that $\mathrm{E}\left(N_{3}\right)=T A U T$. Thus, $N_{3}$ determines the system of tautologies of the classical logic and it is not tautologically many-valued. To verify that, notice that due to the choice of the set of designated elements $\{t, 1\}$, with every $h \alpha \in \operatorname{Hom}(L, A)$ the classical valuation $h^{*} \in \operatorname{Hom}\left(L, M_{2}\right)$ corresponds in a one-to-one way such that $h \alpha \in\{t, 1\}$ iff $h^{*} \alpha=1$.

\section{Structurality and its matrix representation}

The notion of matrix consequence is a natural generalisation of the classical consequence: a relation $\models_{M} \subseteq 2^{F o r} \times$ For is a matrix consequence of $M$ provided that for any $X \subseteq$ For, $\alpha \in$ For $D)$.

$X \models_{M} \alpha$ iff for every $h \in \operatorname{Hom}(L, A)(h \alpha \in D$ whenever $h X \subseteq$ 
It is obvious that $\mathrm{E}(M)=\left\{\alpha: \emptyset \models_{M} \alpha\right\}$. The classical consequence is the matrix consequence of $M_{2}, \models_{M_{2}}$. Therefore, if $M$ determines non-classical set of tautologies then $\models_{M} \neq \models_{M_{2}}$, and the logic identified with the consequence $\models_{M}$ is not many-valued in the second sense. Again, the Eukasiewicz matrix consequence $\models_{M_{3}}$ is a good example. On the other hand, the consequence relation determined by the matrix $N_{3}$ is "entirely" classical, $\models_{N_{3}}=\models_{M_{2}}$.

In the end, let us mention that in the literature one may find examples of matrices, whose content coincides with set of classical tautologies, TAUT, but their consequence relation is non-classical. The matrix $S_{3}$ is one of the kind:

$$
S_{3}=(\{0, t, 1\}, \neg, \rightarrow, \vee, \wedge, \leftrightarrow,\{t, 1\}),
$$

where

\begin{tabular}{c|c}
$\mathrm{x}$ & $\neg \mathrm{x}$ \\
\hline 0 & 1 \\
$\mathrm{t}$ & 1 \\
1 & 0
\end{tabular}

\begin{tabular}{c|ccc}
$\rightarrow$ & 0 & $\mathrm{t}$ & 1 \\
\hline 0 & 1 & 1 & 1 \\
$\mathrm{t}$ & 1 & 1 & 1 \\
1 & 0 & 0 & 1
\end{tabular}

\begin{tabular}{c|ccc}
$\vee$ & 0 & $\mathrm{t}$ & 1 \\
\hline 0 & 0 & 0 & 1 \\
$\mathrm{t}$ & 0 & 0 & 1 \\
1 & 1 & 1 & 1
\end{tabular}

\begin{tabular}{l|lll}
$\wedge$ & 0 & $\mathrm{t}$ & 1 \\
\hline 0 & 0 & 0 & 0 \\
$\mathrm{t}$ & 0 & 0 & 0 \\
1 & 0 & 0 & 1
\end{tabular}

\begin{tabular}{c|ccc}
$\leftrightarrow$ & 0 & $\mathrm{t}$ & 1 \\
\hline 0 & 1 & 1 & 0 \\
$\mathrm{t}$ & 1 & 1 & 0 \\
1 & 0 & 0 & 1
\end{tabular}

Since $\mathrm{t}$ and 0 are indistinguishable by the truth tables in formulas containing the connectives, thus practically in all formulas except the propositional variables, and that both values are distinguished, we obtain $\mathrm{E}\left(S_{3}\right)=T A U T$. Accordingly, $M_{2}$ is the only two-element matrix which might determine $\models_{S_{3}}$. Simultaneously, $\models_{S_{3}} \neq \models_{M_{2}}$, since, for example,

$$
\{p \rightarrow q, p\} \models_{M 2} q \text { while not }\{p \rightarrow q, p\} \models_{M 3} q .
$$

To verify this it simply suffices to turn over a valuation $h$ such that $h p=\mathrm{t}$ and $h q=0$, cf. [7].

The last example shows that it is indispensable to make a distinction between tautological and consequential many-valuedness. Accordingly, the classical system of tautologies was extended to a 
three-valued logic and this extension was assured by rules of inference and not by rejection of logical laws.

The consequence relation has an important algebraic counterpart. With any $\models_{M}$ there may be uniquely associated an operation $\mathrm{Cn}_{M}$ : $2^{\text {For }} \rightarrow 2^{\text {For }}$ such that

$\alpha \in \mathrm{Cn}_{M}(X)$ if and only if $X \models_{M} \alpha$.

$\mathrm{Cn}_{M}$ is called a matrix consequence operation of $M$. The distinguishing property of the logic generated by $M$, i.e. $\models_{M}$ and $C n_{M}$, is its structurality: for any substitution, i.e. an endomorphism of the language, e $\in E N D(L)$,

$$
\begin{aligned}
& X \models_{M} \alpha \text { implies } e X \models_{M} e \alpha, \text { or equivalently } \\
& \alpha \in C n_{M}(X) \text { implies } e \alpha \in C n_{M}(e X) .
\end{aligned}
$$

The concept of structural sentential logic is the ultimate generalisation of the notion of the matrix consequence operation. A structural logic for $L$ is identified with a Tarski's consequence $\mathrm{C}: 2^{\text {For }} \rightarrow 2^{\text {For }}$,

(T0) $X \subseteq \mathrm{C}(X)$

(T1) $\mathrm{C}(X) \subseteq \mathrm{C}(Y)$ whenever $X \subseteq Y$

(T2) $\mathrm{C}(\mathrm{C}(X))=\mathrm{C}(X)$,

satisfying the condition of structurality,

(S) $e \mathrm{C}(X) \subseteq \mathrm{C}(e X)$ for every substitution of $\mathrm{L}$.

Structural logics are characterizable through their set of logical laws and schematic rules of inference. The most important property of these logics is their matrix representation: for every such $\mathrm{C}$ a class of matrices $\underline{\mathrm{K}}$ exists such that $\mathrm{C}$ is the intersection of $\left\{\mathrm{Cn}_{M}: \mathrm{M} \in\right.$ $\underline{\mathrm{K}}\}$ :

$$
\mathrm{C}(X)=\cap\left\{\mathrm{Cn}_{M}(X): M \in \underline{\mathrm{K}}\right\},
$$

for any $X \subseteq$ For.

The property just mentioned is a version of Lindenbaum-Wójcicki completeness theorem, see [12], which was proved using the Lindenbaum bundle $\mathbf{L}$, i.e. the class of all Lindenbaum matrices of the form $(L, C(X)), \mathbf{L}=\{(L, C(X)): X \subseteq$ For $\}$. This fact, when combined 
with the non-Fregean approach by Suszko [10], importantly subscribes to the problem of our interest.

\section{Logical two-valuedness}

The generalization of the truth-functionality principle constituting the base of matrix description of the classical logic is at the heart of the matrix method. Accordingly, the search for common features of the both: many-valued and classical, bivalent logics, is natural. The first serious attempts with this respect can be traced back to early 1950-ies. Especially, to Rosser and Turquette project of formalization of a class of finite-valued standard logics, cf. [9]. The main feature of the sentential part of standard logics was their definitional ability to indicate the counterparts of the classical negation, implication, disjunction, conjunction, and equivalence connectives, which with respect to the division between the undistinguished and distinguished sets of values behave as the classical connectives with respect to the falsity and truth. An important continuation of the idea is due to Bloom and Brown, who in [1] introduced and studied classical abstract logics in which the properties of respective connectives are described by the consequence operation of the logic (the idea of such a characterization may be traced back to Tarski).

Accordingly, in the 1970's the investigations of logical formalizations bore several descriptions of many-valued constructions in terms of zero-one valuations, see e.g. [7]. The approach to the problem by R. Suszko, the author of non-Fregean logic, cf. [10], is the most thoroughly justified. The methodology of this approach will also support our characterization of inferentially many-valued logics.

The base of R. Suszko's philosophy was the distinction between semantic correlates of sentences and their logical values. The author argues that potentially there is no limitation as to the number of correlates of sentences, though there are only two logical values corresponding to Truth and Falsity. In this perspective, all traditionally (i.e. tautologically or consequentially) many-valued logics are also logically two-valued and they may be regarded only as referentially many-valued but not logically many-valued. Recall that the core of the Fregean approach is the so-called Fregean Axiom, see [10], identifying semantic correlates of sentences and their logical values. 
Suszko underlines the referential character of homomorphisms associating sentences with their possible semantic correlates (i.e. referents or situations) and sets them against the logical valuations being zero-one-valued functions on For and, thus, setting apart the referential and the logical valuedness. Suszko claims that each sentential logic, i.e. a structural consequence relation, can be determined by a class of logical valuations and thus, it is logically twovalued. The arguments, supporting the Suszko's thesis, are based on the completeness of any structural consequence $\mathrm{C}$ with respect to a Lindenbaum bundle.

Given a sentential language $L$ and a matrix $M=(A, D)$ for $L$, the set of valuations $\mathrm{TV}_{M}$ is defined as:

$$
\mathrm{TV}_{M}=\left\{\mathrm{t}_{h}: h \in \operatorname{Hom}(L, A)\right\},
$$

where

$$
t_{h}(\alpha)=\left\{\begin{array}{lll}
1 & \text { if } & h(\alpha) \in D \\
0 & \text { if } & h(\alpha) \notin D
\end{array}\right.
$$

Consequently, the matrix consequence operation $\models_{M}$ may be described using valuations as follows:

$$
X \models_{M} \alpha \text { iff for every } t \in T V_{M} t(\alpha)=1 \text { whenever } t(X) \subseteq\{1\} .
$$

The definition of logical valuations may be simply repeated with respect to any structural consequence operation $C$ using its Lindenbaum bundle. Thus, each structural logic $(L, C)$ can be determined by a class of logical valuations of the language $L$ or, in other words, it is logically two-valued.

The justification of the thesis on logical two-valuedness of an important family of logics lacks, however, a uniform description of $T V_{C}$ 's i.e. classes of valuations $C$-adequate, and in each particular case to find $T V_{C}$ is a matter of an elaboration. Below, we discuss the case of finite Łukasiewicz logics.

The original example, given by Suszko, see [11] and [7], presents a relatively easily definable and readable set of logical valuations $L V_{3}$ for the $(\neg, \rightarrow)$ - version of the three-valued Lukasiewicz logic. Accordingly, $L V_{3}$ is the set of all functions $t:$ For $\rightarrow\{0,1\}$ such that for any $\alpha, \beta, \gamma \in F$ or the following conditions hold:

(0) $\mathrm{t}(\gamma)=0$ or $\mathrm{t}(\neg \gamma)=0$ 
(1) $\mathrm{t}(\alpha \rightarrow \beta)=1$ whenever $\mathrm{t}(\beta)=1$

(2) if $\mathrm{t}(\alpha)=1$ and $\mathrm{t}(\beta)=0$, then $\mathrm{t}(\alpha \rightarrow \beta)=0$

(3) if $\mathrm{t}(\alpha)=\mathrm{t}(\beta)$ and $\mathrm{t}(\neg \alpha)=\mathrm{t}(\neg \beta)$, then $\mathrm{t}(\alpha \rightarrow \beta)=1$

(4) if $\mathrm{t}(\alpha)=\mathrm{t}(\beta)=0$ and $\mathrm{t}(\neg \alpha) \neq \mathrm{t}(\neg \beta)$, then $\mathrm{t}(\alpha \rightarrow \beta)=\mathrm{t}(\neg \alpha)$

(5) if $\mathrm{t}(\neg \alpha)=0$, then $\mathrm{t}(\neg \neg \alpha)=\mathrm{t}(\alpha)$

(6) if $\mathrm{t}(\alpha)=1$ and $\mathrm{t}(\beta)=0$, then $\mathrm{t}(\neg(\alpha \rightarrow \beta))=\mathrm{t}(\neg \beta)$

(7) if $\mathrm{t}(\alpha)=\mathrm{t}(\neg \alpha)=\mathrm{t}(\beta)$ and $\mathrm{t}(\neg \beta)=1$, then $\mathrm{t}(\neg(\alpha \rightarrow \beta))=0$.

The uniform description of all finite $L V_{n}$ for $n \geqslant 3$ requires the use of more compound formulas with the well known one-argument connectives $a_{0}, a_{1}, \ldots, a_{n-1}$ of Rosser and Turquette, definable by means of E-implication and E-negation, cf. [9]. Thus, for any $h \in$ $\mathrm{Hom}_{n}$

$$
h\left(a_{i}(\alpha)\right)= \begin{cases}1 & \text { if } h(\alpha)=i / n-1 \\ 0 & \text { otherwise }\end{cases}
$$

Given $n \geqslant 2, L V_{n}$ is then the set of all functions $t \in 2^{F M}$ such that for any $\alpha, \beta, \gamma \in F M$ the following conditions are satisfied:

(1) $t(\gamma)=t\left(a_{n-1}(\gamma)\right) ; t(\neg \gamma)=t\left(a_{0}(\gamma)\right)$.

(2) For each $\gamma \in$ For exactly one of the following equalities holds: $t\left(a_{0}(\gamma)\right)=1$ or $t\left(a_{1}(\gamma)\right)=1 \ldots$ or $t\left(a_{n-1}(\gamma)\right)=1$.

(3) $t\left(a_{i}(\gamma)\right)=1$ or $t\left(\neg a_{i}(\gamma)\right)=1 ; t\left(a_{i}(\neg \gamma)\right)=t\left(a_{n-i-1}(\gamma)\right)$.

(4) $t\left(a_{i}\left(a_{j}(\gamma)\right)\right)= \begin{cases}0 & i \neq 0 \text { and } i \neq n-1 \\ t\left(a_{j}(\gamma)\right) & i=n-1 \\ t\left(\neg a_{j}(\gamma)\right) & i=0 .\end{cases}$

(5) $t(\alpha \rightarrow \beta)=1$ if $t\left(a_{i_{1}}(\alpha)\right)=t\left(a_{i_{2}}(\beta)\right)=1$ and $i_{1} \leqslant i_{2}$, $t\left(a_{i}(\alpha \rightarrow \beta)\right)=1$ if $t\left(a_{i_{1}}(\alpha)\right)=t\left(a_{i_{2}}(\beta)\right)=1$ where $i_{1}>i_{2}$ and $i=(n-1)-i_{1}+i_{2}$.

The last description, originally given and thoroughly justified in [4], is algebraic in its spirit, since the function corresponding to Rosser and Turquette connectives, as well as their counterparts are used in several algebraic formalizations of many-valued logic. This naturally suggests that some other classes of logics may have similar two-valued characterization. 


\section{Inferential three-valuedness}

Logical two-valuedness of any structural logic is a direct consequence of the construction of matrices and the division of the sets $A$ of their elements into two complementary subsets of designated and undesignated elements: $D$ and $A-D$, respectively. And, the $0-1$ valuations are but characteristic functions of the sets of formulas, which are actually associated, via a homomorphism of the language into the matrix, with designated referents. The conclusion that getting out of logical two-valuedness might be made on the basis of a non-complementary division of the set $A$ of referential values.

In [8] a generalisation of Tarski's concept of consequence operation related on the idea that the rejection and acceptance need not be complementary was proposed. The central notions of the framework are counterparts of the concepts of matrix and consequence relation - both distinguished by the prefix " $q$ " which may be read as "quasi". Where $L$ is a sentential language and A is an algebra similar to $L$, a $q$-matrix is a triple

$$
M^{*}=\left(A, D^{*}, D\right),
$$

where $D^{*}$ and $D$ are disjoint subsets of the universe $\mathrm{A}$ of $A\left(D^{*} \cap D=\right.$ $\emptyset) . D^{*}$ are then interpreted as sets of rejected and distinguished elements values of $\mathrm{M}$, respectively. For any such $M^{*}$ one defines the relation $\vdash_{M *}$ between sets of formulae and formulae, a matrix $q$-consequence of $M^{*}$ putting for any $X \subseteq$ For,$\alpha \in F o r$

$X=_{M^{*}} \alpha$ iff for every $h \in \operatorname{Hom}(L, A)\left(h \alpha \in D\right.$ whenever $\left.h X \cap D^{*}=\emptyset\right)$.

The relation of q-consequence was designed as a formal counterpart of reasoning admitting rules of inference which from non-rejected assumptions lead to accepted conclusions. The q-concepts coincide with usual concepts of matrix and consequence only if $D^{*} \cup D=$ $A$, i.e. when the sets $D^{*}$ and $D$ are complementary. Then, the set of rejected elements coincides with the set of non-designated elements.

Sometimes, it is convenient to consider the alternate of $q$-matrix $M^{*}$ received in result of replacing $D^{*}$ with $A-D^{*}$. Then, after renaming we consider the matrix $M_{*}=\left(A, D_{2}, D_{1}\right)$, where $D_{2}=$ 
$A-D^{*}$ and $D_{1}=D$. Note that, then $D_{2} \supseteq D_{1}$. Accordingly,

$X \models_{M^{*}} \alpha$ iff for every $h \in \operatorname{Hom}(L, A)\left(h \alpha \in D_{1}\right.$ whenever $h X \in$ $\left.D_{2}\right)$.

This way of expressing the $q$-consequence will be used in the next Section.

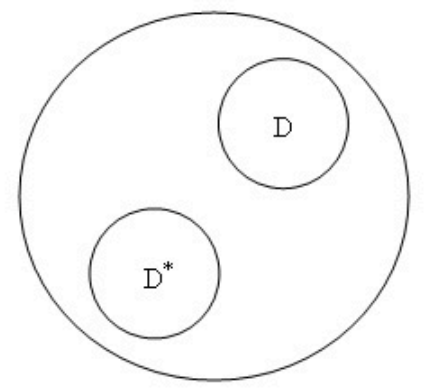

$$
\begin{aligned}
& D^{*} \cap D=\varnothing \\
& D^{*} \cup D \neq A
\end{aligned}
$$

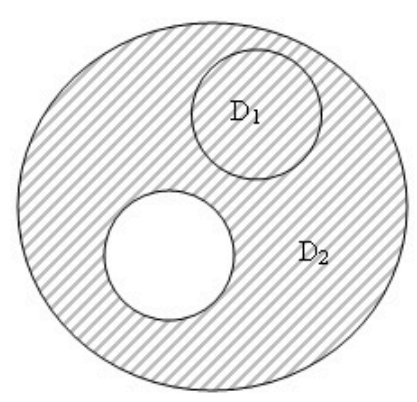

$D_{2} \supseteq D_{1}$

For every $h \in \operatorname{Hom}(L, A)$ a function $k_{h}:$ For $\rightarrow\left\{0,{ }^{1} / 2,1\right\}$

$$
k_{h}(\alpha)=\left\{\begin{array}{lll}
0 & \text { if } & h(\alpha) \in D^{*} \\
1 / 2 & \text { if } & h(\alpha) \in A-\left(D^{*} \cup D\right) \\
1 & \text { if } & h(\alpha) \in D
\end{array}\right.
$$

may be associated. And, given an inference matrix $M^{*}$ for $L$ we put $K V_{M^{*}}=\left\{k_{h}: h \in \operatorname{Hom}(L A)\right\}$, and define $\models_{M^{*}}$ by

$X=_{M^{*}} \alpha \quad$ iff $\quad$ for every $k_{h} \in \mathrm{KV}_{M^{*}}$ if $k_{h}(X) \subseteq\{1 / 2,1\}$, then $k_{h}(\alpha)=1$.

With each relation $\models_{M^{*}}$ one may associate the operation $W n_{M^{*}}$ : $2^{\text {For }} \rightarrow 2^{\text {For }}$ putting

$$
W n_{M^{*}}(X)=\left\{\alpha: X \models_{M^{*}} \alpha\right\} .
$$

This is a kind of a three-valued description of $\left.\right|_{M^{*}}$. Notice that $K V_{M^{*}}$ reduces to $T V_{M}$ and $\models_{M^{*}}$ to $\models_{M}$ when $D^{*} \cup D=A$. It is worth emphasising that this is a case only exceptionally: for the 
ordinary (structural) consequence relation. In general, the description remains three-valued. Therefore a q-logic $\left(L, W n_{M^{*}}\right)$ is either two- or three-valued.

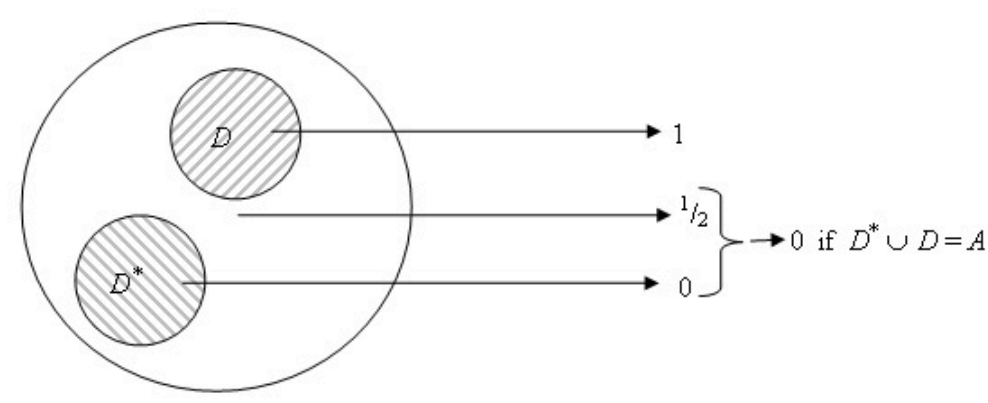

The following example proves that the three-valued q-logics exist, cf. [8]. Take the three-element q-matrix

$$
\mathrm{E}_{q}^{3}=(\{0,1 / 2,1\}, \neg, \rightarrow, \vee, \wedge, \leftrightarrow,\{0\},\{1\}),
$$

where the connectives are defined as in the Lukasiewicz three-valued logic. Then, for any $p \in V a r$, it is not true that $\{p\} \vdash_{M^{*}} p$. To see this, it suffices to consider the valuation sending $p$ into $1 / 2$.

The more striking is perhaps the fact that even logics generated by some two-element q-matrices may be logically three-valued.

Though different, the two approaches coincide on contents. Note, that

For any q-matrix $M^{*}=\left(A, D^{*}, D\right)$ and a corresponding matrix $M=(A, D), W n_{M^{*}}(\emptyset)=C n_{M}(\emptyset)=E(M)$.

This is a very important observation. It means that any logical system may equally well be extended to logically two-valued logic $\left(L, C n_{M}\right)$ or to a three-valued logic $\left(L, W n_{M^{*}}\right)$. Obviously, depending on the quality and cardinality of $M$ the two kinds of extensions may define different logics. Moreover, in several cases it is also possible to define two (or more) different inferential extensions of a given system. The idea was applied in [5] to make a distinction between two "indistinguishable" modal connectives of the four-valued modal system of Łukasiewicz. 
The $q$-framework is general. In [8] an inference operation of which $W n_{M^{*}}$ is a prototype was introduced and studied. An operation $W: 2^{\text {For }} \rightarrow 2^{\text {For }}$ is a $q$-consequence operation provided that for every $X, Y \subseteq$ For

(W2) $W(X) \subseteq W(Y)$ whenever $X \subseteq Y$.

$W$ is called structural if for any substitution $e \in \operatorname{End}(\mathcal{L})$

$$
e W(X) \subseteq W(e X) .
$$

Where $M^{*}$ is any inference matrix, $W n_{M^{*}}$ is structural. In turn, all Lindenbaum's tools may be adopted to structural inference operations $W$ to exactly the same effect. Thus, the bundle of Lindenbaum's inference matrices

$$
W_{X}=(\text { For }, \text { For }-(X \cup W(X)), W(X))
$$

may be used to prove, cf.[8], that

For every structural inference operation $W$ there is a class $\underline{K}$ of inferential matrices such that

$$
W n_{K}(X)=\bigcap\left\{W n_{M^{*}}(X): M^{*} \in \underline{K}\right\} .
$$

Following the line of constructing the valuations corresponding to the classes of matrices, here applied to q-matrices, we may get a necessary description of any structural q-logic $(L, W)$. Accordingly, we conclude that any such construction is logically two- or threevalued.

\section{$5 \quad$ More inferential values?}

Extending the inferential approach to more values and thus getting logical $n$-valuedness for $n>3$ seems easy: apply the method of construction described in Section 4 and divide matrix universe into more than three mutually disjoint subsets. However, the next step, i.e. construction of an appropriate matrix $q(n)$-consequence is not evident. On the other hand, no natural lattice-theoretic combination of q-consequences is possible, since $q$-consequence operations on a given language form a lattice, see [6]. 
In [3] a partial solution of the problem is provided. Using special bundle of finite linear matrices with the unary functions "labelling" appropriate subsets, the construction of a compositional operation was defined, which in the "inferential" terms is more than 3 valued.

The construction is based on a finite algebra $\underline{E}_{n}=\left(E_{n}, f_{1}, f_{2}, \ldots\right.$, $f_{m}$ ) where $n \geq 2$ and $E_{n}=\{1,2,3, \ldots, n\}$. Next, it is required that among the functions of $\underline{E}_{n}$, primitive or definable, there are unary functions $\delta_{1}, \delta_{2}, \ldots, \delta_{n}:^{2}$

$$
\delta_{i}(x)=\left\{\begin{array}{lll}
n & \text { if } & x \geq i+1 \\
1 & \text { if } & x<i+1
\end{array}\right.
$$

Let $\mathrm{L}=\left(E_{n}, F_{1}, F_{2}, \ldots, F_{m}\right)$ be the language to which $\underline{E}_{n}$ is similar. Assume that $D_{k}=\{k, k-1, \ldots, n\}$ and $D_{l}=\{l$, $l+1, \ldots, n\}$, for $k<l$ and, finally take the $q$-matrix: ${ }^{3}$

$$
M_{k, l}^{o}=\left(\underline{E}_{n}, D_{k}, D_{l}\right),
$$

Consider its $q$-inference,

$X \vdash M_{k, l}^{o} \alpha$ iff for every $h \in \operatorname{Hom}\left(\underline{L}, \underline{E}_{n}\right)$ (If $h(X) \subseteq D_{k}$ then $\left.h(\alpha) \in D_{l}\right)$,

and the matrix $M^{n}=\left(\underline{E}_{n},\{n\}\right)$ and its consequence $\models_{M}^{n}$

$X \models_{M}^{n} \alpha$ iff for every $h \in \operatorname{Hom}\left(\underline{L}, \underline{E}_{n}\right)$ (If $h(X) \subseteq\{n\}$, then $h(\alpha) \in\{n\})$.

Let $\delta_{k}(X)=d f\left\{\delta_{k}(\beta): \beta \in X\right\}$ and notice that

$$
X \vdash_{M_{k, l}}^{o} \alpha \text { iff } \delta_{k}(X) \models_{M}^{n} \delta_{l}(\alpha),
$$

and, finally, that

$$
Q n_{M_{k, l}}(X)=\left\{\alpha: X \vdash_{M_{k, l}}^{o} \alpha\right\}=\left\{\alpha: \delta_{k}(X) \models_{M}^{n} \delta_{l}(\alpha)\right\} .
$$

The last equation shows two descriptions of sets $Q n_{M_{k, l}}(X)$ and thus, descriptions of the matrix $q$-consequence operation $Q n_{M_{k, l}}$.

\footnotetext{
${ }^{2}$ Such functions are definable in standard logics, cf. [9], a family of which contains most of the known finite-valued calculi. They are also widely used in modern formulations of Post algebras.

${ }^{3}$ In the second formulation provided in Section 4.
} 
The crucial concept of a multidimensional matrix and its inference is defined using a decreasing sequence of non-empty subsets of $E_{n}$, $D_{s-1}, \ldots, D_{1}$ :

$$
D_{s-1} \supseteq D_{s-2} \supseteq \ldots \supseteq D_{1} \text {, }
$$

such that $E_{n}-D_{s-1} \neq \emptyset$.

The structure

$$
\mathbf{M}_{s}=\left(\underline{E}_{n}, D_{s-1}, D_{s-2}, \ldots, D_{1}\right)
$$

is referred to as an $s$ dimensional q-matrix. The notion of an $s$ dimensional $q$-matrix is a direct generalization of the concept of $q$ matrix; every $q$-matrix is three dimensional and "standard" matrices are two dimensional. Next to that, as compatible with the appropriate consequence-like operation $Q^{s}$ defined below, the partitions correspond directly to logical values, see [3] for details.

The notion of $s$ dimensional $q$-matrix inference which would keep the correspondence preserved to a higher degree is then defined using the bundle $\mathbf{M}^{\mathbf{s}}$ of $q$-matrices associated with $\mathbf{M}_{\mathbf{s}}$ :

$$
\mathbf{M}^{\mathbf{s}}=\left\{\left(E_{n}, D_{s-1}, D_{s-2}\right),\left(E_{n}, D_{s-2}, D_{s-3}\right), \ldots,\left(\underline{E}_{n}, D_{2}, D_{1}\right)\right\},
$$

or, equivalently, by

$$
\mathbf{M}^{\mathbf{s}}=\left\{M_{s-2}, M_{s-3}, \ldots, M_{1}\right\},
$$

where $M_{s-i}={ }_{d f}\left(\underline{E}_{n}, D_{s-i+1}, D_{s-i}\right), i=1, \ldots, s-2$.

Now, if $Q_{i}=W n_{M_{s-i}}$ is the matrix $q$-consequence of $M_{s-i}$, then the operation $Q^{s}: 2^{\text {For }} \rightarrow 2^{\text {For }}$ defined as superposition of $Q_{s-2}$, $Q_{s-3}, \ldots, Q_{2}, Q_{1}, Q^{s}=Q_{s-2} \circ Q_{s-3} \circ \ldots \circ Q_{2} \circ Q_{1}$, which for every set of formulas $X$ :

$$
Q^{s}(X)=Q_{s-2}\left(Q_{s-3}\left(\ldots\left(Q_{2}\left(Q_{1}(X)\right)\right) \ldots\right)\right)
$$

satisfies the starting requirements.

The definition of multiple-element valuations is slightly involved. To outline the idea of it, fully discussed in [3], we shall consider the case of 5-dimensional matrix q-consequence $W$ determined by a matrix $\mathbf{M}_{5}=\left(\underline{E}_{n}, D_{4}, D_{3}, D_{2}, D_{1}\right)$. 
As one may expect, the logic determined by $W$ should be inferentially five-valued. One may also expect that, as in the threevalued case of q-consequence, the valuations should be in correspondence with homomorphisms from $L$ into $E_{n}$, i.e. the referential assignments sending formulas into the matrix universe. Accordingly, valuations are functions mapping appropriate subsets of $E_{n}$ into the five-valued set of logical (inferential) values, say into $\left\{0,1 / 4,{ }^{2} / 4,{ }^{3} / 4,1\right\}$. The subsets of $E_{n}$, mapped in subsequent values are respectively: $E_{n}-D_{4}, D_{4}-D_{3}, D_{3}-D_{2}, D_{2}-D_{1}, D_{1}$. The following diagram

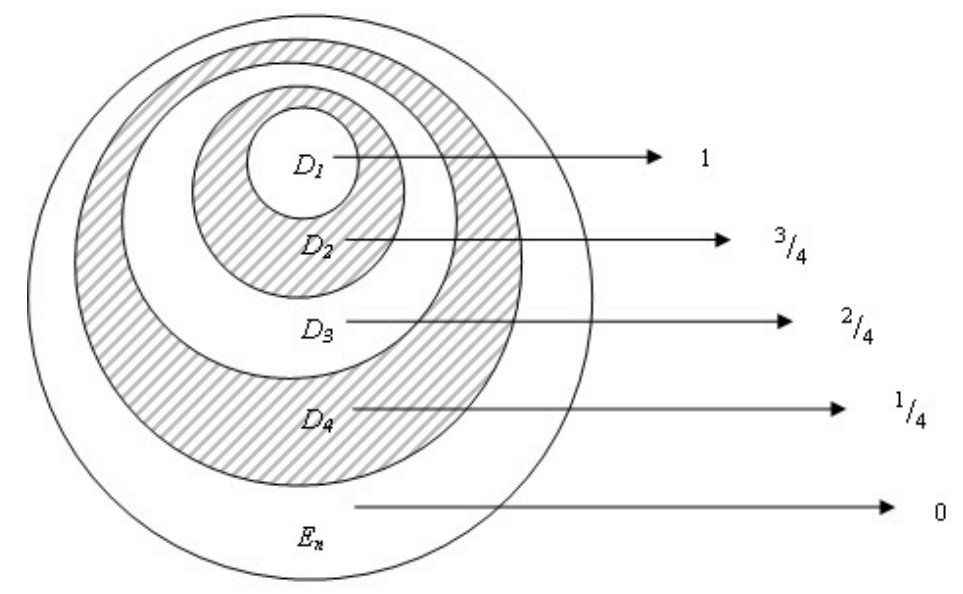

depicts the association.

\section{Final remarks}

The modern conceptual treatment of many-valuedness was possible due to matrices introduced to logic on the turn of 20 th century. The matured matrix methodology, as a part of a general theory of logical calculi, cf. [12], essentially extended the possibilities.

In the paper we discussed two kinds of matrix based referential many-valuedness: tautological and consequential. The first case occurs, when the multiplication of elements of matrix universe results in a smaller set of logical truths, i.e. set properly included in the set of all classical tautologies, see Section 1. Otherwise, it is possible to get many-valuedness using the matrix consequence: conservatively, saving the classical set of tautologies, or non-conservatively. The first case requires the use of "non-classical rules" of inference, see Section 2. 
The concept of many-valuedness becomes more involved on the grounds of the theory of structural consequence operations, since logics thus defined are often characterizable with the classes of matrices, cf. [12]. So, sometimes it may be difficult to say to what degree a given logic is (referentially, or algebraically) "many-valued". The situation essentially changes in view of the Suszko's methodology inspired by the non-Fregean approach in which denotations of sentences i.e. referential, or algebraic values are different entities from logical values. Accordingly, due to the Suszko's Thesis, every structural logic is logically two-valued.

The construction of $q$-consequence was the first step towards logical many-valuedness, or more precisely, to inferential three-valuedness. The methodological background for structural $q$-logics has already been elaborated: the q-consequence is described through modification of Tarski's conditions for consequence and the $q$-matrix methodology as a generalisation of the standard matrix approach, is provided. Finally, the Lindenbaum-Wójcicki's - like completeness result leads to the conclusion that any structural $q$-logic is logically two- or three-valued. Accordingly, the logical three-valuedness departs naturally from the division of the matrix universe into three subsets and the $(S T)$ counterpart says that any inference based on a structural $q$-consequence may have a bivalent or a three-valued description, cf. Section 3.

The discussion of possibilities for further exploration of the idea of logical $n$-valuedness for $n>3$ showed that the only evident step to be made consists of a division of the matrix universe into more than three subsets. Therefore, instead of the general approach to logically many-valued inference, only finite linear matrices with oneargument functions "labelling" subsets of elements of the matrix were considered. Unfortunately, even for the case, which is important, getting uniform description of properties of logically morevalued consequence-like relation is not easy. So, one may only say, that the construction presented in Section 4 proves that logically $n$-valued logics do exist. The problem of getting general description and elaboration of methodology for such devices still remains open.

It should be also underlined, that getting a general axiomatization of structural s-dimensional q-consequence, as well as its completeness proof, seems very hard indeed. Perhaps the algebraic hints given 
in Section 3 may help in making further steps toward even more general theory of many-valued inference.

\section{References}

[1] Bloom, S. L., Brown D. J. Classical abstract logics, Dissertationes Matematicae, CII, 1973, 43-52.

[2] Eukasiewicz, J. O logice trójwartościowej, Ruch Filozoficzny, 5, 1920, 170-171. English tr. On three-valued logic [in:] Borkowski, L. (ed.) Selected works, NorthHolland, Amsterdam, 87-88.

[3] Malinowski, G. 'Beyond three inferential values', Studia Logica, 92, 2009, 203-213.

[4] Malinowski, G. Classical characterization of n-valued Łukasiewicz calculi, Reports on Mathematical Logic, 9, 1977, 41-45.

[5] Malinowski, G. 'Inferential intensionality', Studia Logica, 76, 2004, 3-16.

[6] Malinowski, G. 'Lattice properties of a protologic inference', Studies in Logic, Grammar and Rhetoric, 4 (17), Language, Mind and Mathematics, 2001, 51-58.

[7] Malinowski, G. Many-valued logics, Oxford Logic Guides, 25, Clarendon Press, Oxford, 1993.

[8] Malinowski, G. Q-consequence operation, Reports on Mathematical Logic, 24, 1990, 49-59.

[9] Rosser, J. B., Turquette, A. R. Many-valued logics, North-Holland, Amsterdam, 1952.

[10] Suszko, R. Abolition of the Fregean Axiom [in:] Parikh, R. (ed.) Logic Colloquium, Symposium on Logic held at Boston, 1972-73, Lecture Notes in Mathematics, vol. 453, 1972, 169-239.

[11] Suszko, R. The Fregean Axiom and Polish Mathematical Logic in the 1920s, Studia Logica, XXXVI (4), 1977, 377-380.

[12] Wójcicki R. Theory of logical calculi. Basic theory of consequence operations, Synthese Library, 199. Kluwer Academic Publishers, Dordrecht, 1988. 